2. HAO Y, HONG X, ZHAO X. Congenital absence of the portal vein associated with focal nodular hyperplasia of the liver and congenital heart disease (Abernethy malformation): A case report and literature review. Oncology Letters. 2015;9:695-700.

3. Howard ER, Davenport M. Congenital extrahepatic portocaval shunts: the Abernethy malformation. J Pediatr Surg 1997;32:494-7.

4. Morgan G, Superina R. Congenital absence of the portal vein: two cases and a proposed classification system for portasystemic vascular anomalies. J Pedi $\neg$ atr Surg 1994;29:1239-41.

5. Murray CP, Yoo SJ, Babyn PS. Congenital extrahe $\neg$ patic portosystemic shunts. Pediatr Radiol 2003;33:614-20.

6. Emre S, Arnon R, Cohen E, Morotti RA, Vaysman D, Shneider BL. Resolution of hepatopulmonary syndrome after auxiliary partial orthotropic liver transplantation in Abernethy malformation: a case report. Liver Transpl 2007;13:1662-8.

7. Gallego C, Miralles M, Marin C, Muyor P, Gonzales G, Garcia-Hidalgo E. Congenital hepatic shunts. RadioGraphics 2004;24:755-72

8. Blanc T, Guerin F, Franchi- Abella $\mathrm{S}$ et al. Congenital portosystemic shunts in children ; A new anatomical classification correlated with surgical strategy. Ann Surg 2014;260:188-98.

\section{Spontaneous Large Esophageal Lesion with Upper GI Bleed}

Intramural hematoma of the esophageal (IHE) is an uncommon esophageal injury most frequently described in anticoagulated elderly patients and often confused with other benign or malignant esophageal, cardiac, pulmonary or aortic lesions. It usually has a benign course and selfresolves within 3 weeks. We present a case of an elderly patient who underwent stent assisted coiling of ruptured basilar artery aneurysm (BAA) with peri-procedural coverage of antiplatelets and anticoagulation, and post intervention developed a large spontaneous intramural esophageal hematoma.

\section{Case Report}

A 65 year old lady presented at our center with history of sudden onset headache and vomiting followed by loss of consciousness, raising suspicion of subarachnoid hemorrhage which was confirmed on non-contrast CT scan of the head. Subsequently CT angiography of intracranial and neck vessels revealed a ruptured Basilar Artery aneurysm $(5 \times 4.9 \times 4.5 \mathrm{~mm})$ for which she underwent digital subtraction angiography with stent assisted coiling of the aneurysm. Periprocedural dual anti platelet medication (ecosprin and clopidogrel) and anticoagulation (intravenous heparin infusion) were startedas per protocol, and Ryle's tube was placed. Her sensorium gradually improved over the next 48 hours. Two days after the procedure, she had upper gastrointestinal bleed with a hemoglobin drop of $3.5 \mathrm{gm} / \mathrm{dl}$. She was stabilized with blood transfusion and intravenous proton pump inhibitor (PPI) infusion. Emergency esophageduodenoscopy showed a large bluish lesion extending from upper esophagus ( $23 \mathrm{~cm}$ from incisor) to gastro-esophageal junction (Figure 1). CT chest revealed a large and long segment intramural lesion of the esophagus (Figure 2). Based on the clinical presentation of the patient and typical endoscopic findings, diagnosis of a large spontaneous intramural esophageal hematoma was made. Anticoagulation and clopidogrel were stopped and she was maintained on ecosprin, anti-hypertensives, and PPIs.

Repeat endoscopy at 1 week showed reduction of the size of the lesion (Figure 3), and patient was started orally and subsequently discharged. At 3 weeks, endoscopy showed complete resolution of the lesion with a long healing ulcer (Figure 4). 


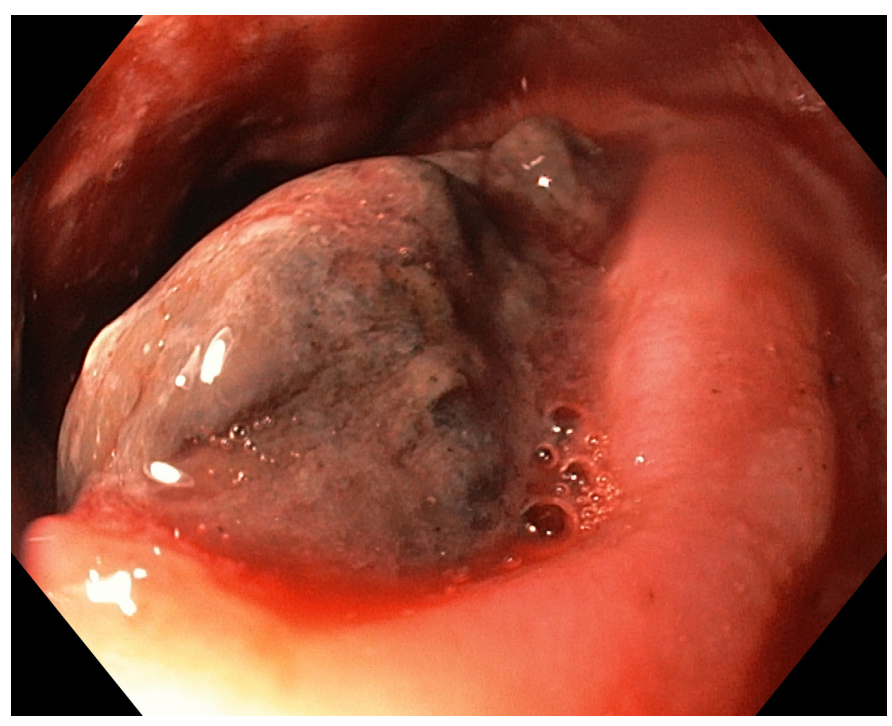

Figure 1: Esophageduodenoscopy showing a large bluish lesionin the esophagus.

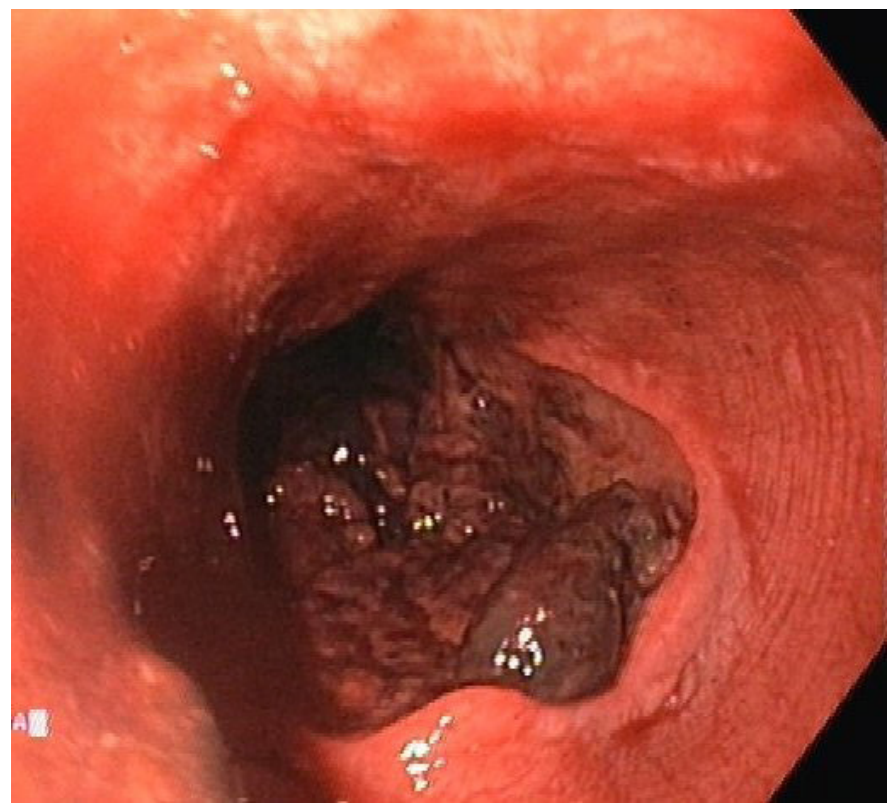

Figure 3: Endoscopic image (after 1 week) showing reduction in size of esophageal hematoma.

\section{Discussion}

Esophageal intramural hematoma ${ }^{1}$, also known as "intramural rupture of the esophagus" or "esophageal apoplexy" is a rare esophageal injury, characterized by bleed between esophageal mucosa and muscularispropria, involving a long segment of the esophagus. Hematemesis

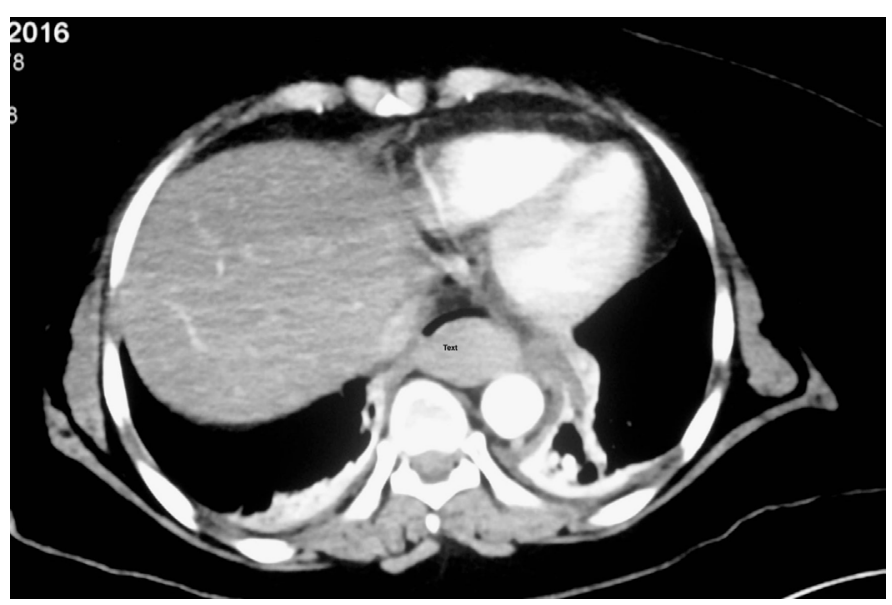

Figure 2: CT chest with intravenous contrast showing a large and long segment intramural non-enhancing lesion of the esophagus.

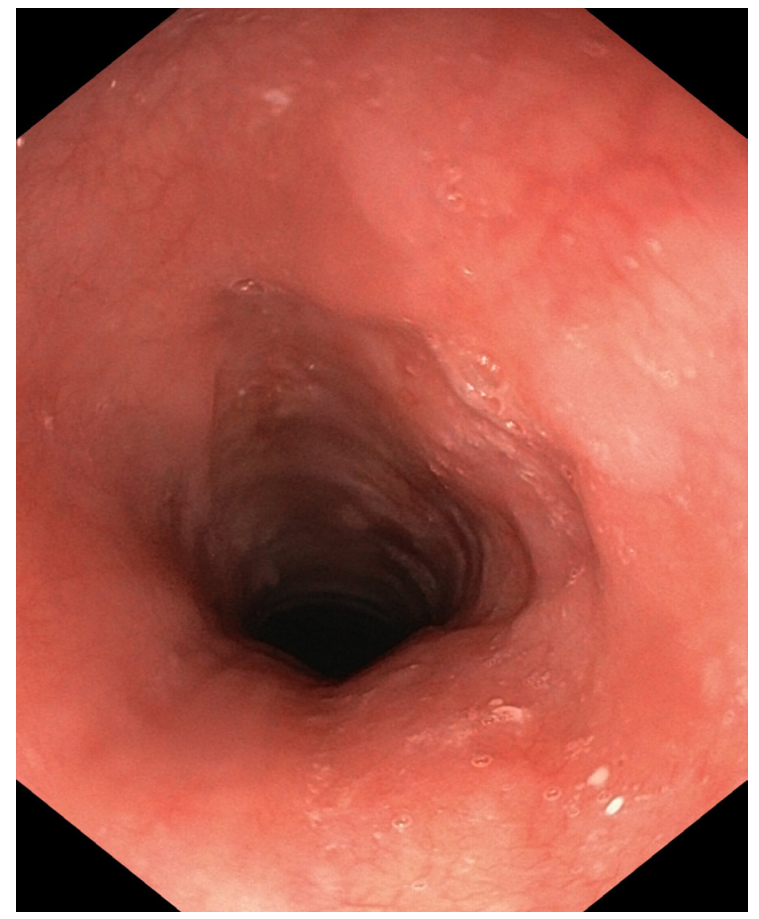

Figure 4: Endoscopic image (after 3 week) showing complete resolution of esophageal hematoma with an overlying ulcer.

occurs later due to breach of the mucosa. A review of 174 cases has shown that it occurs more commonly in elderly females on anticoagulation, with F:M ratio of 1.8:1 and average age of 58.8 years. $^{2}$ of these, 103 case reports had described spontaneous occurrence with underlying predisposing conditions such as cirrhosis, hemodialysis, 
coagulation disorders, and patients on anticoagulants and antiplatelets. Etiologies proposed for this uncommon condition include suboptimal hemostasis, emetogenic, traumatic, aortic disease related and idiopathic. $36 \%$ patients present with the triad of retrosternal chest pain (due to esophageal distension by expanding hematoma), hematemesis and dysphagia or odynophagia, with $80 \%$ reporting at least 2 of these symptoms. ${ }^{3}$ Dyspnoea is unusual but may occur from tracheal compression by the hematoma. In our case, the use of anticoagulants was the predisposing factor, with hematoma occurring either spontaneously or secondary to nasogastric tube insertion. Repeated suctioning of nasogastric tube may produce negative pressure to the esophageal mucosa, leading to submucosal injury and consequent hematoma. ${ }^{3}$

The most common site for hematoma is the distal esophagus $(83 \%)$ followed by the middle esophagus (78\%) and proximal esophagus (27\%). Contrast swallow (barium or gastrograffin) typically shows a "double barrel esophagus" or "mucosal stripe" sign, and CT scan with intravenous contrast shows thickened esophageal wall with a non-enhancing, high-attenuation intramural mass causing luminal compromise. ${ }^{5}$ Endoscopy typically shows an intraluminal protruded mass covered by fluctuant purple or dark-colored mucosa running along the long axis of esophagus, that may be intact or show a laceration. Endoscopic ultrasound shows a uniform low echo mass in the submucosal layer, and can also be useful to assess other submucosal lesions and mediastinal structures. Treatment is mainly conservative with reversal of anticoagulation, parenteral nutrition and analgesics. $96 \%$ hematomas resolve spontaneously over a period of 1 to 3 weeks 1 , with only $2.3 \%$ deaths reported.

Hence, intramural hematoma of the esophagus is an unusual condition resulting from heamorrhage within the esophageal wall with a threatening presentation. It is important to differentiate it from other esophageal conditions (particularly malignancies) due to its benign course and lack of need of therapeutic intervention. Recognition of this condition by clinical presentation, imaging and endoscopic finding should prevent clinicians from inappropriate diagnosis and treatment.
NEHA BERRY NARENDRA DHAKA SAROJ KANT SINHA RAKESH KOCHHAR

Department of Gastroenterology, Postgraduate Institute of Medical Education and Research, Chandigarh, India-160012

Corresponding Author: Dr Narendra Dhaka Email:dhakanaren@gmail.com

\section{References}

1. Cheung J, Müller N \& Weiss A. Spontaneous intramural esophageal hematoma: case report and review. Can. J. Gastroenterol. 2006;20:285-6.

2. Nagai $T$, Torishima R, Nakashima H, Uchida A, Okawara $\mathrm{H}$, Suzuki K, et al. Spontaneous esophageal submucosal hematoma in which the course could be observed endoscopically. Intern Med. 2004;43:461-7.

3. Fujimoto Y, Shirozu K, Shirozu N, Akiyoshi K, Nishimura A, Kawasaki Set al.Esophageal Submucosal Hematoma Possibly Caused by Gastric Tube Insertion Under General Anesthesia.A A Case Rep. 2016;15:169-171.

4. Cao DT, Reny JL, Lanthier N, Frossard JL. Intramural Hematoma of the Esophagus. Case Rep. Gastroenterol.2012;6:510-7.

5. Restrepo CS, Lemos DF, Ocazionez D, Moncada R, Gimenez CR. Intramural hematoma of the esophagus: a pictorial essay. Emerg Radiol 2008;15:13-22.

\section{Acute Cytopenias after Liver Transplantation: A Case of Thrombotic Thrombocytopenic Purpura}

Thrombotic thrombocytopenic purpura (TTP) is a rare disease and diagnosis requires high index of suspicion. Timely diagnosis is very important as mortality is very 